[2] J. Bourgain, Fourier Transform Restriction Phenomena for Certain Lattice Subsets and Applications to Nonlinear Evolution Equations, Geometric and Functional Analysis 3 (1993), 107-156.

[3] M. Christ, J. Colliander and T. Tao, Instability of the periodic nonlinear Schrödinger equation, Preprint, 2003.

[4] M. Hochbruck, A. Ostermann, Explicit exponential Runge-Kutta methods for semilinear parabolic problems SIAM J. Numer. Anal. 43 (2005), 1069-1090,

\title{
Numerical Methods for Multiscale Problems
}

\section{Assyr Abdulle}

Consider $u^{\varepsilon}$, the solution of a (partial) differential equation

$$
L^{\varepsilon} u^{\varepsilon}=f^{\varepsilon},
$$

where $L^{\varepsilon}$ is a linear differential operator for which $\varepsilon$ indicates the small scales in the coefficients. Due to the oscillations in these coefficients, $u^{\varepsilon}$ will typically exhibit small scale oscillations. The scale gap between the length of the oscillation and the phenomena of interest (large scale behavior) makes direct simulation of (1) with a standard numerical method often impossible. A common approach is to compute an effective or homogenized operator $\bar{L}$, in which the small scales have been averaged out and to solve the corresponding homogenized equation (see [9] and references therein)

$$
\bar{L} \bar{u}=\bar{f} .
$$

The problem (2) can be solved with standard methods. However, this procedure has several drawbacks. First, restrictive assumptions on the data (periodicity, homogeneity) are needed to derive explicit equations for the homogenized problem 2 . Second, the coefficients of the homogenized equation have usually to be computed numerically so that a control of the overall procedure (i.e. the numerical discretization of the homogenized equations with numerically computed coefficients) is difficult. Third, the fine scale behavior, i.e. the oscillations of the solution, is lost in the homogenization process.

In this report we discuss several numerical methods for multiscale problems constructed within the framework of the Heterogeneous Multiscale Methods (HMM) [1], [2], [3], [4], [5], [6], [7], [8]. These methods discretize the physical problem directly by a "macroscopic numerical model" with a macroscopic discretization. The input coefficients of the macroscopic numerical model are unknown, since the macroscopic model is not supposed to be known (we do not precompute an averaged equation as (2)). These coefficients are recovered on the fly by solving a "microscopic numerical model" on sampling domains within the macro discretization. A variety of micro-macro approaches based on iterative schemes, have been proposed in the literature, mainly for elasticity problems, including nonlinear problems (see [5] for a discussion and references). The iterative procedure in these approaches increases the cost of the methods and makes a full error control of these micro-macro schemes difficult even for linear problems. 
Heterogeneous multiscale methods for variational problems. We consider the following elliptic model problem in the domain $\Omega \subset \mathbb{R}^{d}$

$$
-\nabla \cdot\left(a^{\varepsilon} \nabla u^{\varepsilon}\right)=f \text { in } \Omega, \quad u^{\varepsilon}=0 \text { on } \partial \Omega,
$$

where we assume that the tensor $a^{\varepsilon}(x)=a\left(x, \frac{x}{\varepsilon}\right)=a(x, y)$ is symmetric, coercive and periodic with respect to each component of $y$ in the unit cube $Y=(0,1)^{d}$. We further assume that $f \in L^{2}(\Omega), a_{i j}(x, \cdot) \in L^{\infty}\left(\mathbb{R}^{d}\right)$, that $x \rightarrow a_{i j}(x, \cdot)$ is smooth from $\bar{\Omega} \rightarrow L^{\infty}\left(\mathbb{R}^{d}\right)$ and that $\Omega$ is a convex polygon. The FE-HMM for the elliptic homogenization problem, based on the macro finite element $(\mathrm{FE})$ space $S_{0}^{1}\left(\Omega, \mathcal{T}_{H}\right)$ $\left(\mathcal{T}_{H}\right.$ is the macro triangulation) is defined by a modified macro bilinear form [3],[8],

$$
B\left(u^{H}, v^{H}\right)=\sum_{K \in \mathcal{T}_{H}} \frac{|K|}{\left|K_{\varepsilon}\right|} \int_{K_{\varepsilon}} \nabla u^{h} a\left(x_{k}, x / \varepsilon\right)\left(\nabla v^{h}\right)^{T} d x,
$$

where $K_{\varepsilon}=x_{k}+\varepsilon[-1 / 2,1 / 2]^{d}$ is a sampling sub-domain centered at the barycenter $x_{k}$ of the triangle $K$, where $|K|,\left|K_{\varepsilon}\right|$ denote the measure of $K$ and $K_{\varepsilon}$, respectively, and where $u^{h}$ is the solution of the following micro problem: find $u^{h}$ such that $\left(u^{h}-u^{H}\right) \in W_{p e r}^{1}\left(K_{\varepsilon}\right)$ and

$$
\int_{K_{\varepsilon}} \nabla u^{h} a\left(x_{k}, x / \varepsilon\right)\left(\nabla z^{h}\right)^{T} d x=0 \quad \forall z^{h} \in S_{p e r}^{1}\left(K_{\varepsilon}, \mathcal{T}_{h}\right) .
$$

For $K_{\varepsilon} \subset K \in \mathcal{T}_{H}$, we consider the micro FE space $S_{\text {per }}^{1}\left(K_{\varepsilon}, \mathcal{T}_{h}\right) \subset H_{\text {per }}^{1}\left(K_{\varepsilon}\right) / \mathbb{R}$ of piecewise linear polynomials on the micro triangulation $\mathcal{T}_{h}$, periodic on the boundary $\partial K_{\varepsilon}$. The meshsizes of the macro and micro spaces are denoted by $H$ and $h$, respectively. The following convergence estimates have been obtained in [3] for the fully discrete FE-HMM

$$
\begin{aligned}
& \left\|u^{0}-u^{H}\right\|_{H^{1}(\Omega)} \leq C\left(H+\left(\frac{h}{\varepsilon}\right)^{2}\right), \quad\left\|u^{0}-u^{H}\right\|_{L^{2}(\Omega)} \leq C\left(H^{2}+\left(\frac{h}{\varepsilon}\right)^{2}\right), \\
& \left\|u^{\varepsilon}-u_{p}^{\varepsilon}\right\|_{\bar{H}^{1}(\Omega)} \leq C\left(H+\varepsilon+\frac{h}{\varepsilon}\right),
\end{aligned}
$$

where $u^{\varepsilon}$ is the solution of problem (3), $u^{0}$ is the solution of the homogenized problem corresponding to $(3)$ and $u_{p}^{\varepsilon}$ is a reconstructed solution obtained from $u^{H}$ with fine scale solution $\left(u-u^{H}\right)$ periodically extended on each element $K$ (see [8],[1]). For the estimate (7), the norm $\bar{H}$ is mesh dependent since $u_{p}^{\varepsilon}$ can be discontinuous across the macro elements $K$. Similar fully discrete estimates have also be derived for the effective velocity in transport problems [4] and for elasticity problems [5].

Heterogeneous multiscale methods for dynamic problems. Consider the parabolic homogenization problem

$$
\begin{aligned}
& \frac{\partial u^{\varepsilon}}{\partial t}=\nabla \cdot\left(a^{\varepsilon} \nabla u^{\varepsilon}\right) \text { in }(0, T) \times \Omega \\
& u^{\varepsilon}=0 \quad \text { on }(0, T) \times \partial \Omega, \quad u^{\varepsilon}(0, x)=g(x) \text { on } \Omega,
\end{aligned}
$$

where $u^{\varepsilon}=u^{\varepsilon}(t, x), \Omega \subset \mathbb{R}^{d}$ is a bounded domain, and $a^{\varepsilon}(x)$ is symmetric, uniformly coercive and bounded. We further assume that $a^{\varepsilon}(x)$ and $g(x)$ are regular 
enough in order to have a smooth solution of the above problem. In the sequel we describe the algorithm in one dimension for simplicity (see [1] for generalizations). A coarse model for (8) is given by $\frac{\partial U}{\partial t}=\nabla \cdot\left(a^{0} \nabla U\right)$, where $a^{0}$ (the homogenized tensor) reflects the large scale impact of $a^{\varepsilon}$. As for the previous problem, we do not assume that $a^{0}$ is known. The finite difference heterogeneous multiscale method (FD-HMM) [1] is defined by a macroscopic scheme on a coarse grid $\left\{x_{i}\right\}_{i=0}^{N}$ of $\Omega$

$$
U_{i}^{k+1}=U_{i}^{k}+\Delta t \frac{J_{i+1 / 2}^{k}+J_{i-1 / 2}^{k}}{\Delta x}
$$

with meshsize $\Delta x$ and evolved with a coarse time step $\Delta t$. The unknown fluxes $J_{i \pm 1 / 2}=\frac{ \pm 1}{\left|K_{x_{i \pm 1 / 2}}^{\varepsilon}\right|} \int_{K_{x_{i \pm 1 / 2}}^{\varepsilon}} a^{\varepsilon} \nabla \hat{u}^{\varepsilon} d x$ are given by the average of micro solutions $\hat{u}^{\varepsilon}$ obtained by solving small scale problems on sampling domains $K_{x_{i \pm 1 / 2}}^{\varepsilon}$ of size $\varepsilon$ centered around $x_{i \pm 1 / 2}$ for a small time $\left[t^{k}, t^{k}+\delta\right]$

$$
\begin{aligned}
& \frac{\partial \hat{u}^{\varepsilon}}{\partial t}=\nabla \cdot\left(a^{\varepsilon} \nabla \hat{u}^{\varepsilon}\right)(t, x) \in\left(t^{k}, t^{k}+\delta\right) \times K_{x_{i \pm 1 / 2}}^{\varepsilon}, \\
& \hat{u}^{\varepsilon}-U^{k}(x) \quad \varepsilon \text {-periodic on }\left(t^{k}, t^{k}+\delta\right) \times \partial K_{x_{i \pm 1 / 2}}^{\varepsilon}, \quad \hat{u}\left(t^{k}, x\right)=U^{k}(x),
\end{aligned}
$$

where $\delta$ is a relaxation time (see [1]). The initial values at the cells $K_{x_{i \pm 1 / 2}}^{\varepsilon}$ are obtained by linear reconstruction of the known solution $\left\{U_{i}^{k}\right\}_{i=0}^{N}$ at time $t^{k}$.

For the convergence analysis [1] we have to introduce an intermediate problem. Let $\left\{U_{i}^{k}\right\}_{i=0}^{N}$ be the solution of the FD-HMM at time $t^{k}=t_{0}+k \Delta t$ and let $\left\{\bar{U}_{i}^{k}\right\}_{i=0}^{N}$ be the solution of a finite difference method similar to (10) but with fluxes $\bar{J}_{i \pm 1 / 2}=\left(a^{0}\left(\bar{U}_{i \pm 1 / 2}-\bar{U}_{i}\right)\right) / \Delta x$. Then it can be shown [1]

$$
\max _{0 \leq i \leq N}\left|U_{i}^{k}-\bar{U}_{i}^{k}\right| \leq C T \frac{\varepsilon}{\Delta x} \quad \forall t_{k} \in[0, T] .
$$

$\left\{\bar{U}_{i}^{k}\right\}_{i=0}^{N}$ can be seen as a standard FD method for the homogenized problem, for which standard convergence results, provided enough smoothness of the solution, give $\max _{i}\left|U\left(t^{k}, x_{i}\right)-\bar{U}_{i}^{k}\right| \leq C T\left(\Delta t+(\Delta x)^{2}\right) \quad \forall t_{k} \in[0, T]$. Combining both results, we obtain the following error estimate for the FD-HMM when compared to the homogenized solution of problem (8)

$$
\max _{0 \leq i \leq N}\left|U\left(t^{k}, x_{i}\right)-U_{i}^{k}\right| \leq C T\left(\Delta t+(\Delta x)^{2}+\frac{\varepsilon}{\Delta x}\right) \quad \forall t_{k} \in[0, T] .
$$

Here, the exact solution for the micro problem (11) has been assumed. For a fully discrete analysis, the discretization of this problem with a micro spatial mesh with meshsize $\delta \xi$ has to be considered. The term $\frac{\delta \xi}{\varepsilon}$ is expected to appear [6].

\section{REFERENCES}

[1] A. Abdulle and W. E, Finite difference HMM for homogenization problem, J. Comput. Phys. 191 (2003), pp. 18-39.

[2] A. Abdulle and C. Schwab, Heterogeneous multiscale FEM for diffusion problem on rough surfaces, SIAM Multiscale Model. Simul. 3 (2005), pp. 195-220.

[3] A. Abdulle, On a-priori error analysis of fully discrete heterogeneous multiscale FEM, SIAM Multiscale Model. Simul. 4 (2005), pp. 447-459. 
[4] A. Abdulle, Multiscale methods for advection- diffusion problems, Discrete Contin. Dyn. Syst. B, Suppl. Vol. (2005), pp. 11-21.

[5] A. Abdulle, Analysis of a heterogeneous multiscale FEM for problems in elasticity, Math. Mod. Meth. Appl. Sci. (M3AS) 16 (2006), pp.1-21.

[6] A. Abdulle, Finite volume heterogeneous multiscale method, in preparation.

[7] W. E and B. Engquist, The heterogeneous multiscale methods, Comm. Math. Sci. 1 (2003) pp. 87-132.

[8] W. E, P. Ming and P. Zhang, Analysis of the heterogeneous multi-scale method for elliptic homogenization problems, J. Amer. Math. Soc. 18 (2004), pp. 121-156.

[9] V.V. Jikov, S.M. Kozlov and O.A. Oleinik, Homogenization of differential operators and integral functionals, Springer-Verlag, Berlin, Heidelberg, 1994.

\section{Structural Preserving Isospectral Flows for Quadratic Pencils Moody T. Chu \\ (joint work with Nicoletta Del Buono)}

The eigeninformation $(\lambda, \mathbf{u})$ of the quadratic pencil,

$$
Q(\lambda):=Q\left(\lambda ; M_{0}, C_{0}, K_{0}\right)=\lambda^{2} M_{0}+\lambda C_{0}+K_{0},
$$

is critical to the understanding of the dynamical system

$$
M_{0} \ddot{\mathbf{x}}+C_{0} \dot{\mathbf{x}}+K_{0} \mathbf{x}=f(t),
$$

which arises frequently in many important applications, including applied mechanics, electrical oscillations, vibro-acoustics, fluid mechanics, and signal processing. It is easy to see that the linear pencil,

$$
L(\lambda):=L\left(\lambda ; M_{0}, C_{0}, K_{0}\right)=\left[\begin{array}{cc}
C_{0} & M_{0} \\
M_{0} & 0
\end{array}\right] \lambda+\left[\begin{array}{cc}
K_{0} & 0 \\
0 & -M_{0}
\end{array}\right],
$$

in the so called Lancaster structure, is equivalent to $Q(\lambda)$. Recently, it has been shown that for almost all quadratic pencils there exists real-valued $2 n \times 2 n$ real matrices $\Pi_{\ell}$ and $\Pi_{r}$ such that

$$
\Pi_{\ell}^{\top} L(\lambda) \Pi_{r}=L\left(\lambda ; M_{D}, C_{D}, K_{D}\right)=\left[\begin{array}{cc}
C_{D} & M_{D} \\
M_{D} & 0
\end{array}\right] \lambda+\left[\begin{array}{cc}
K_{D} & 0 \\
0 & -M_{D}
\end{array}\right],
$$

where $M_{D}, C_{D}, K_{D}$ are all real-valued $n \times n$ diagonal matrices $[2,3,4]$. Such a transformation is significant in that it links the dynamical behavior of a multipledegree-of-freedom system directly to that of a system consisting of $n$ independent single-degree-of-freedom subsystems. It breaks down the interlocking connectivity in the original system into totally disconnected subsystems while preserving the entire spectral properties. Thus it will be of great value in practice if the transformations $\Pi_{\ell}$ and $\Pi_{r}$ can be found from any given pencil. The theory of existence of $\Pi_{\ell}$ and $\Pi_{r}$ in $[2,3]$ was established on the basis of the complete spectral information of $L(\lambda)$. To construct $\Pi_{\ell}$ and $\Pi_{r}$ from the availability of spectral information certainly is impractical. The emphasis of this talk is to construct $\Pi_{\ell}$ and $\Pi_{r}$ numerically by structure preserving isospectral flows without knowing the spectral information. 